\title{
Musgos (Bryophyta) de um fragmento de Mata Atlântica na Serra da Jibóia, município de Santa Terezinha, BA, Brasil
}

\author{
Emilia de Brito Valente ${ }^{1,4}$, Kátia Cavalcanti Pôrto ${ }^{1}$, Silvana Brito Vilas Bôas-Bastos ${ }^{2,3}$ e Cid José Passos Bastos ${ }^{3}$
}

Recebido em 21/11/2007. Aceito em 30/07/2008

\begin{abstract}
RESUMO - (Musgos (Bryophyta) de um fragmento de Mata Atlântica na Serra da Jibóia, município de Santa Terezinha, BA, Brasil). O levantamento de musgos realizado em uma área de Mata Atlântica no município de Santa Terezinha, Bahia, resultou em flora rica, com 61 espécies pertencentes a 23 famílias e 46 gêneros. Sematophyllaceae (sete spp.), Orthotrichaceae (seis spp.), Pilotrichaceae (cinco spp.), Calymperaceae (cinco spp.), Leucobryaceae (cinco spp.) e Meteoriaceae (quatro spp.) apresentaram maior riqueza específica. Actinodontium integrifolium (Broth.) Churchill e Calymperes venezuelanum (Mitt.) Broth. ex Pittier constituem novos registros para o Brasil. Ectropothecium leptochaeton (Schwaegr.) W.R. Buck, Eulacophyllum cultelliforme (Sull.) W.R. Buck \& Ireland, Fissidens santaclarensis Thér., Lepidopilidium portoricense (Müll. Hal.) H.A. Crum \& Steere, Mittenothamnium reptans (Hedw.) Card., Orthostichella pentasticha (Brid.) W.R. Buck, Pilotrichella flexilis (Hedw.) Ångstr., Porotrichum mutabile Hampe e Thuidium tomentosum Schimp. são novas ocorrências para a Bahia. Houve predomínio de táxons de distribuição neotropical. A comunidade corticícola foi predominante com $70 \%$ das espécies, seguida da epíxila, com 23\%. A brioflora do fragmento mostrou-se rica já que corresponde a 24\% do total de briófitas atualmente conhecido no Estado.
\end{abstract}

Palavras-chave: briófitas, brioflora, comunidades, floresta submontana, musgos

\begin{abstract}
Mosses (Bryophyta) from a fragment of Atlantic Forest in the Jibóia Mountains, Santa Terezinha municipality, Bahia State, Brazil). This work presents the results of an inventory of mosses carried out in an Atlantic Forest fragment in the Jibóia Mountains, Santa Terezinha, Bahia State, Brazil. A total of 61 moss species distributed in 23 families and 46 genera were found. The families Sematophyllaceae (seven spp.), Orthotrichaceae (six spp.), Pilotrichaceae (five spp.), Calymperaceae (five spp.), Leucobryaceae (five spp.) and Meteoriaceae (four spp.) had higher species richness. Actinodontium integrifolium (Broth.) Churchill and Calymperes venezuelanum (Mitt.) Pitt. are new records for Brazil. Ectropothecium leptochaeton (Schwaegr.) W.R. Buck., Eulacophyllum cultelliforme (Sull.) W.R. Buck \& Ireland, Fissidens santa-clarensis Thér., Mittenothamnium reptans (Hedw.) Card., Orthostichella pentasticha (Brid.) Buck, Pilotrichella flexilis (Hedw.) Ångstr., Lepidopilidium portoricense (Müll. Hal.) Crum \& Steere, and Thuidium tomentosum Schimp. are new records for Bahia. As regards geografic distribuition, neotropical taxa predominate, while in relation to communities, corticicolous (70\%) and epixilous species (23\%) predominate. The local flora was considered rich for including $24 \%$ of the bryophytes that occur in Bahia State.
\end{abstract}

Key words: bryophytes, bryoflora, communities, mosses, submontane forest

\section{Introdução}

A flora de musgos da Bahia totaliza, atualmente, ca. 270 espécies, se compiladas as citações dos trabalhos de Bastos et al. (1998a; 1998b; 2000), Bastos \& Vilas Bôas-Bastos (1998), Bastos \& Yano (1993; 2006), Vilas Bôas-Bastos \& Bastos (1998), Yano \& Bastos (1994) realizados para formações de restinga, campo rupestre, caatinga e área urbana, bem como os "checklists" de Yano (1981; 1989a; 1995; 1996a; 2004; 2006a; 2006b).

$\mathrm{O}$ trabalho tem como objetivo contribuir para o aumento do conhecimento da flora de musgos de remanescentes de Mata Atlântica, ainda pouco explorados no Estado da Bahia, e vem complementar o levantamento da brioflora da Serra da Jibóia, realizado por Valente \& Pôrto (2006a; 2006b), que relataram para a área de mata da encosta, 70 espécies de hepáticas (Marchantiophyta), entre as quais se destacaram várias tipicamente epífilas (que ocorrem preferencialmente sobre folhas) que são indicadoras de ambientes em bom estado de preservação, e vários novos registros para o Nordeste e Bahia. Também foi realizado um inventário brioflorístico no afloramento rochoso situado no topo da Serra que resultou em 21 espécies, sendo 11 hepáticas e 10 musgos,

\footnotetext{
1 Universidade Federal de Pernambuco, Departamento de Botânica, Centro de Ciências Biológicas, Programa de Pós-Graduação em Biologia Vegetal, Av. Professor Moraes Rêgo s.n., Cidade Universitária, 50760-420 Recife, PE, Brasil

2 Universidade Estadual de Feira de Santana, Programa de Pós-Graduação em Botânica

3 Universidade Federal da Bahia, Instituto de Biologia, Departamento de Botânica, Laboratório de Taxonomia de Briófitas, Campus de Ondina, 40170-280 Salvador, BA, Brasil

4 Autor para correspondência: ebvalente@gmail.com
} 
com dominância de musgos acrocárpicos, rupícolas e heliófilos (Valente \& Pôrto 2006c).

Estudos sobre a brioflora da Serra da Jibóia são de relevância fundamental já que área representa uma das 147 prioritárias para a conservação da Mata Atlântica, sendo considerada de extrema importância biológica (MMA 2002).

\section{Material e métodos}

Área de estudo - A serra da Jibóia localiza-se na latitude $12^{\circ} 51^{\prime} \mathrm{S}$ e longitude $39^{\circ} 28^{\prime} \mathrm{W}$, tem altitude compreendida entre 400-800 msnm, extensão de 6 km, e está inserida no município de Santa Terezinha, Estado da Bahia. Apresenta em suas encostas Floresta Ombrófila Densa Montana de acordo com a classificação de Veloso \& Góes Filho (1982), vegetação rupestre no topo e caatinga na base (Queiroz et al. 1996). Caracterização mais detalhada da área pode ser encontrada em Sobrinho \& Queiroz (2005) e Valente \& Pôrto (2006b).

Amostragem e estudo do material - Seis excursões foram realizadas entre os anos de 2001 e 2004, abrangendo todas as estações do ano. O material foi coletado e herborizado de acordo com Yano (1989b), e posteriormente depositado no Herbário da Universidade Estadual de Feira de Santana (HUEFS). As amostras foram coletadas sobre troncos vivos e em decomposição, rocha, folhas e solo. As identificações foram baseadas principalmente na seguinte literatura: Florschütz (1964); Costa (1986); Frahm (1991); Sharp et al. (1994); Yano (1996b); Yano \& Oliveira-e-Silva (1997); Buck (1998); Gradstein et al. (2001); Reese (1993); Visnadi (2002); Vilas Bôas-Bastos \& Bastos (2004); Vaz \& Costa (2006a; 2006b).

A literatura utilizada para a obtenção da distribuição geográfica no mundo e no Brasil foi a seguinte: Yano (1981; 1989a; 1995; 1996a; 2004; 2006a; 2006b); Visnadi (2005; 2006); Peralta \& Yano (2006); Vaz \& Costa (2006a; 2006b); Yano \& Pôrto (2006); Campelo \& Pôrto (2007).

As siglas dos estados brasileiros estão de acordo com o Instituto Brasileiro de Geografia e Estatística IBGE.

O sistema de classificação adotado foi baseado em Goffinet \& Buck (2004).

\section{Resultados e discussão}

O levantamento resultou em 61 espécies de musgos pertencentes a 23 famílias e 46 gêneros (Tab. 1). Duas espécies são novos registros para o Brasil, Actinodontium integrifolium e Calymperes venezuelanum. Actinodontium integrifolium caracteriza-se pelos caulídios eretos, filídios oblongos e agudos a curto-acuminados, com margens recurvadas da base até quase o ápice, seta lisa (Fig. 1-5) e peristômio densamente papiloso. Está geograficamente distribuida no norte da América do Sul (Venezuela) e América Central; ocorre como epífita e saxícola em florestas úmidas a ca. 500-1.100 m de altitude (Buck 1998). Calymperes venezuelanum é facilmente reconhecida por seu hábito graminiforme, filídios rigidamente eretos a flexuosos, longos e quebradiços, interrompidos acima das cancelinas por um intervalo de costa nua, semelhante a um pecíolo (Fig. 6-13). Espécie rara, tem distribuição no norte da América do Sul e América Central, hábito terrícola e epífita e ocorre em florestas a ca. 300-900 m de altitude (Reese 1993).

Pseudotrachypus martinicensis está sendo citada pela segunda vez para o Brasil, tendo sido o primeiro registro também para uma área de Mata Atlântica do estado da Bahia (S.B.Vilas-Bôas Bastos, dados não publicados). Esta espécie se caracteriza por possuir costa simples, filídios com margens conspicuamente onduladas e células unipapilosas, apresenta distribuição mundial semelhante à das duas espécies citadas acima, ocorrendo apenas no México e América Central, sobre galhos, em florestas úmidas a ca. 500-2.000 m de altitude (Buck 1998).

Nove espécies constituem novos registros para o Estado da Bahia: Ectropothecium leptochaeton, Eulacophyllum cultelliforme, Fissidens santa-clarensis, Lepidopilidium portoricense, Mittenothamnium reptans, Orthostichella pentasticha, Pilotrichella flexilis, Porotrichum mutabile e Thuidium tomentosum. As famílias que apresentaram maior riqueza específica foram Sematophyllaceae (sete spp.), Orthotrichaceae (seis spp.), Pilotrichaceae (cinco spp.), Calymperaceae (cinco spp.), Leucobryaceae (cinco spp.) e Meteoriaceae (quatro spp.), que se encontram entre as mais representativas em florestas tropicais (Richards 1984; Gradstein \& Pócs 1989; Gradstein et al. 2001).

O padrão de distribuição geográfica mundial das espécies é predominantemente neotropical (53\% do total). Algumas, contudo, têm distribuição mais restrita, a exemplo de Barbula riograndensis, endêmica do Brasil, e outras endêmicas da América do Sul como Syrrhopodon lanceolatus (Colômbia, Brasil e Suriname) e Floribundaria flaccida (Colômbia e Brasil).

As comunidades corticícola (70\%) e epíxila (23\%) predominaram sobre as terrícola $(10 \%)$, rupícola $(8 \%)$ e epífila (5\%). Estes resultados eram esperados, já que em florestas tropicais, troncos vivos e em decomposição são, nesta ordem, os substratos mais disponíveis e preferenciais para os musgos. Aliado a isso está a escassez de rochas na área de estudo e a grande quantidade de serapilheira, que dificulta o estabelecimento de briófitas sobre o solo. 
Tabela 1. Espécies de musgos ocorrentes na Serra da Jibóia, município de Santa Terezinha, BA, Brasil, com dados sobre substrato e distribuição geográfica mundial e no Brasil. Comunidades: $\mathrm{CO}=$ corticícola, EF = Epífila, EX = Epíxila, TE = Terrícola, $\mathrm{RU}=\mathrm{Rupícola}$. * = novo registro para a Bahia $\mathrm{e}^{* *}=$ novo registro para o Brasil.

\begin{tabular}{|c|c|c|c|}
\hline Espécies & Comunidade & $\begin{array}{c}\text { Distribuição geográfica } \\
\text { mundial }\end{array}$ & $\begin{array}{c}\text { Distribuição geográfica } \\
\text { no Brasil }\end{array}$ \\
\hline
\end{tabular}

\section{BRACHYTHECIACEAE \\ Brachythecium ruderale (Brid.) Buck Squamidium brasiliense (Hornsch.) Broth. \\ S. leucotrichum (Taylor) Broth. \\ BRYACEAE \\ Bryum argenteum \\ B. grandifolium (Taylor) Müll. Hal. \\ B. coronatum Schwägr. \\ B. huillense Welw. \& Duby \\ CALYMPERACEAE \\ Calymperes venezuelanum (Mitt.) Pitt.** \\ Octoblepharum albidum Hedw.}

$\begin{array}{cc}\mathrm{CO} & \text { Neotrópico e Sul dos EUA } \\ \mathrm{CO} & \text { Neotrópico e África } \\ \mathrm{CO} / \mathrm{EF} & \text { Neotrópico }\end{array}$

$\begin{array}{cc}\text { TE } & \text { Ampla } \\ \text { EX/TE/CO } & \text { Neotrópico } \\ \text { RU } & \text { Sul dos EUA, Neotrópico e Ásia } \\ \text { CO/TE } & \text { Neotrópico e África } \\ \text { CO } & \text { Neotrópico } \\ \text { CO } & \text { Ampla }\end{array}$

Syrrhopodon lanceolatus (Hampe) W.D. Reese EX

S. parasiticus (Sw. ex Brid.) Paris

S. prolifer var. prolifer Schwägr.

DICRANACEAE

Holomitrium arboreum Mitt.

Leucoloma serrulatum Brid.

FISSIDENTACEAE

Fissidens santa-clarensis Thér.*

HOOKERIACEAE

Crossomitrium patrisiae (Brid.) Müll. Hal.

HYPNACEAE

Ectropothecium leptochaeton (Schwägr.) W.R. Buck*

Mittenothamnium reptans (Hedw.) Cardot*

Vesicularia vesicularis var. portoricensis (Brid.) W.R. Buck

HYPOPTERYGIACEAE

Hypopterygium tamariscinum (Hedw.) Brid.

LEMBOPHYLLACEAE

Orthostichella pentastica (Brid.) W.R. Buck*

Pilotrichella flexilis (Hedw.) Angström*

LEUCOBRYACEAE

Bryohumbertia filifolia var. humilis (Mont.) J.-P. Frahm

Campylopus arctocarpus (Hornsch) Mitt.

Leucobryum albidum (Brid. ex P. Beauv.) Lindb.

L. crispum Müll. Hal.

L. martianum (Hornsch.) Hampe ex Müll. Hal. EX

\section{METEORIACEAE}

Aerobryopsis plumaria (Hampe) M. Fleisch.

Floribundaria flaccida (Mitt.) Broth.

Meteorium deppei (Hornsch. ex Müll. Hal.) Mitt.

Pseudotrachypus martinicensis (Broth.) W.R. Buck

$\mathrm{CO}$

$\mathrm{CO}$
$\mathrm{CO}$

$\mathrm{CO}$

$\mathrm{CO}$

$\mathrm{EF}$
América do Sul Sul dos EUA e Pantropical

Neotrópico e África

EX Neotrópico

CO Netrópico

CO/EX Neotrópico

CO Neotrópico

EX Neotrópico

CO Neotrópico e África

EX Neotrópico

CO/EX Ampla

CO Neotrópico

EF Neotrópico e África

CO Neotrópico

EX/CO Sul dos EUA, Neotrópico e África EX Ampla

CO Neotrópico

Neotrópico

Neotrópico Colômbia e Brasil Neotrópico

Neotrópico
ES, MS, PE, PR, RS

BA, ES, MG, PE, RJ, RS, SP AC, AL, AM, BA, ES, MG, PE, PR, RJ, RR, RS, SC, SP

AL, AM, BA, CE, DF, ES, GO, MG, MT, PB, PE, PR, RJ, RR, RS, SC, SP RJ, RR, SC, SP, BA, MT, RO AC, AM, BA, DF, FN, GO, MA, MG, MT, PA, PE, PR, RJ, RO, RR, RS, SC, SP BA, MT, RJ, RO, SP, PR, SC, SP

Primeira citação para o Brasil AC, AL, AM, AP, BA,CE, DF, ES, FN, GO, MA, MG, MS, MT, PA, PB, PE, PI, PR, RJ, RN, RO, RR, SC, SE, SP, TO AM, BA, CE, PA, PB, PR, RJ, SC, SP AC, AL, AM, BA, CE, ES, MG, MS, MT, PA, PI, PE, PR, RJ, RO, RR, RS, SC, SE, SP AL, AM, BA, CE, DF, ES, GO, MG, MT, PA, PE, PI, PR, RJ, RO, RS, SC, SE, SP, TO

AM, BA, MG, PA, PE, RJ, RO, RR, SP AL, BA, ES, MG, PE, RJ, SP

PA

AC, AM, BA, PA, PE, RJ, RO, RR

AM, ES, MG, MT, MS, PA, PR, RJ, SC

ES, PE, PR, SC, RS, MT, RJ, MG BA, MT, MS

RJ, SP

AM, DF, ES, MG, PE, RJ, RO, RS, SC, SP ES, MG, PE, PR, RJ, RS, SC e SP

AM, BA, CE, MG, PE, PR, RJ, RR, RS, SC, SP

BA, MT, MG, PR, RS, RJ, SC, SP BA, MT, MG, PA, RJ, SP

AM, AP, BA, DF, ES, GO, MG, MT, PR, RJ, RR, RS, SC, SP AC, AM, AP, BA, CE, ES, MA, MG, MT, PA, PE, PR, RJ, RR, RO, SC, SE, SP

BA, MG, MT, RJ, RS, SC, SP BA, ES, MG, PE, PR, RJ, RS, SC, SP BA, CE, ES, MS, PE, PR, RJ, SP BA 


\begin{tabular}{|c|c|c|c|}
\hline Espécies & Comunidade & $\begin{array}{c}\text { Distribuição geográfica } \\
\text { mundial }\end{array}$ & $\begin{array}{c}\text { Distribuição geográfica } \\
\text { no Brasil }\end{array}$ \\
\hline \multicolumn{4}{|l|}{ NECKERACEAE } \\
\hline Porotrichum mutabile Hampe* & $\mathrm{CO}$ & Neotrópico & PE, RJ, RS \\
\hline \multicolumn{4}{|l|}{ ORTHOTRICHACEAE } \\
\hline Macromitrium cirrosum (Hedw.) Brid. & $\mathrm{CO}$ & Neotrópico & AP, BA, PA, PR, RJ, SC, SP \\
\hline M. punctatum (Hook. \& Grev.) Brid. & EX & Neotrópico & AM, BA, CE, ES, GO, RJ \\
\hline M. richardii Schwägr. & $\mathrm{CO}$ & Sul dos EUA, África, Neotrópico & AM, BA, ES, MG, PR, RJ, RS, SC, SP \\
\hline $\begin{array}{l}\text { Groutiella apiculata (Hook.) H.A. Crum \& } \\
\text { Steere }\end{array}$ & $\mathrm{CO}$ & Neotrópico & BA, ES, MT, MS, PA, PE, PR, RJ, SP \\
\hline Schlotheimia rugifolia (Hook.) Schwägr. & $\mathrm{CO} / \mathrm{EX}$ & EUA e Neotrópico & $\begin{array}{l}\text { AC, AM, BA, CE, DF, ES, GO, MG, } \\
\text { MT, PA, PE, PR, RJ, RO, RS, SC, SP }\end{array}$ \\
\hline Zygodon reinwardtii (Hornsch.) A. Braun & $\mathrm{CO}$ & Neotrópico e Ásia & ES, BA, RJ, MG, SC, SP \\
\hline \multicolumn{4}{|l|}{ PHYLLOGONIACEAE } \\
\hline Phyllogonium viride Brid. & $\mathrm{CO}$ & Neotrópico & AL, BA, ES, MG, PE, PR, RJ, RS, SC, SP \\
\hline \multicolumn{4}{|l|}{ PILOTRICHACEAE } \\
\hline $\begin{array}{l}\text { Actinodontium integrifolium (Broth.) } \\
\text { S.P. Churchill** }\end{array}$ & $\mathrm{CO}$ & Neotrópico & Primeira citação para o Brasil \\
\hline Hypnella pallescens (Hook.) A. Jaeger & EX & Neotrópico & AM, BA, MT, PA, RJ \\
\hline Lepidopilum scabrisetum (Schwägr.) Steere & $\mathrm{CO}$ & Neotrópico & AM, BA, MT, RJ \\
\hline $\begin{array}{l}\text { Lepidopilidium portoricense (Müll. Hal.) } \\
\text { H.A. Crum \& Steere* }\end{array}$ & $\mathrm{CO}$ & Neotrópico & $\mathrm{SC}, \mathrm{SP}$ \\
\hline Thamniopsis incurva (Hornsch.) W.R. Buck & EX & Neotrópico & AM, BA, PA, PB, RJ, RS, SP \\
\hline \multicolumn{4}{|l|}{ PYLAISIADELPHACEAE } \\
\hline Taxithelium planum (Brid.) Mitt. & RU/TE & Sul dos EUA, Neotrópico, África & $\begin{array}{c}\text { AC, AL, AM, AP, BA, ES, GO, MG, MR, } \\
\text { MA, MT, MS, PA, PB, PE, PR, RJ, RO, } \\
\text { RR, SC, SP, TO }\end{array}$ \\
\hline \multicolumn{4}{|l|}{ POLYTRICHACEAE } \\
\hline Polytrichum commune Hedw. & TE & Ampla & AM, BA, GO, MG, PR, RJ, RR, RS, SC \\
\hline \multicolumn{4}{|l|}{ POTTIACEAE } \\
\hline Barbula riograndensis E.B. Bartram & TE & Brasil & $\mathrm{BA}, \mathrm{RJ}, \mathrm{RS}$ \\
\hline Hyophila involuta (Hook.) A. Jaeger & RU & Ampla & $\begin{array}{c}\text { AM, BA, CE, DF, ES, GO, MS, MT, PA, } \\
\text { PB, PE, PI, PR, RJ, RO, RS, SP }\end{array}$ \\
\hline \multicolumn{4}{|l|}{ RACOPILACEAE } \\
\hline Racopilum tomentosum (Hedw.) Brid. & RU & Neotrópico e Sul dos EUA & $\begin{array}{l}\text { AC, AM, BA, CE, DF, ES, GO, MG, MT, } \\
\text { MS, PA, PE, PR, RJ, RO, RS, SP, SC }\end{array}$ \\
\hline \multicolumn{4}{|l|}{ RHIZOGONIACEAE } \\
\hline Pyrrhobryum spiniforme (Hedw.) Mitt. & $\mathrm{CO} / \mathrm{EX}$ & Ampla & $\begin{array}{c}\text { AM, BA, ES, MG, MT, PE, PR, RJ, RO, } \\
\text { RR, RS, SC, SP }\end{array}$ \\
\hline \multicolumn{4}{|l|}{ SEMATOPHYLLACEAE } \\
\hline $\begin{array}{l}\text { Acroporium estrellae (Müll. Hal.) W.R. Buck \& } \\
\text { Schäfer-Verw. }\end{array}$ & $\mathrm{CO}$ & Neotrópico & BA, CE, MG, PA, PR, RJ, RS, SC, SP \\
\hline A. longisrostre (Brid.) Buck & $\mathrm{CO}$ & Neotrópico & $\mathrm{AM}, \mathrm{BA}, \mathrm{PE}, \mathrm{RJ}$ \\
\hline A. pungens (Hedw.) Broth. & $\mathrm{CO}$ & Neotrópico & AP, AM, BA, ES, MG, PR, RJ, RR, SC, SP \\
\hline Sematophyllum adnatum (Michx.) E. Britton & $\mathrm{CO}$ & Ampla & AM, BA, CE, DF, ES, MT, PA, RJ, SP \\
\hline S. galipense (Müll. Hal.) Mitt. & $\mathrm{CO}$ & Neotrópico & $\begin{array}{c}\text { BA, CE, ES, GO, MG, MT, PA, PE, PR, RJ, } \\
\text { RO, RR, RS, SC, SP, TO }\end{array}$ \\
\hline S. subpinnatum (Brid.) E. Britton & $\mathrm{CO}$ & Ampla & $\begin{array}{l}\text { AC, AM, AP, BA, CE, DF, ES, GO, MG, } \\
\text { MS, MT, PA, PE, RJ, RO, RR, SC, SP }\end{array}$ \\
\hline S. subsimplex (Hedw.) Mitt. & $\mathrm{CO}$ & Neotrópico & $\begin{array}{l}\text { AC, AM, AP, BA, DF, ES, GO, MA, MG, } \\
\text { MT, MS, PA, PB, PE, PI, PR, RJ, } \\
\text { RO, RR, RS, SE, SC e SP }\end{array}$ \\
\hline \multicolumn{4}{|l|}{ STEREOPHYLLACEAE } \\
\hline $\begin{array}{l}\text { Entodontopsis leucostega (Brid.) W.R. Buck \& } \\
\text { Ireland }\end{array}$ & $\mathrm{CO}$ & Neotrópico e Ásia & $\begin{array}{c}\text { AC, AM, BA, CE, DF, FN, GO, MA, MG, } \\
\text { MS, MT, PA, PB, PE, PI, RO, RJ, } \\
\text { RO, RR, SC, SP, TO }\end{array}$ \\
\hline $\begin{array}{l}\text { Eulacophyllum cultelliforme (Sull.) } \\
\text { W.R. Buck \& Ireland* }\end{array}$ & $\mathrm{CO}$ & Neotrópico & AM, ES, MG, MS, MT, PB, PE, PR, RJ, SE, SP \\
\hline Pilosium chlorophyllum (Hornsch.) Müll. Hal. & $\mathrm{CO}$ & Neotrópico & $\begin{array}{l}\text { AC, AL, AM, AP, BA, CE, ES, GO, MG, } \\
\text { MS, MT, PA, PE, RJ, RO, RR, SP, TO }\end{array}$ \\
\hline \multicolumn{4}{|l|}{ THUIDIACEAE } \\
\hline Thuidium tomentosum Schimp.* & RU & Neotrópico & BA, ES, MS, MT, PE, PR, RJ, RR, SP \\
\hline
\end{tabular}



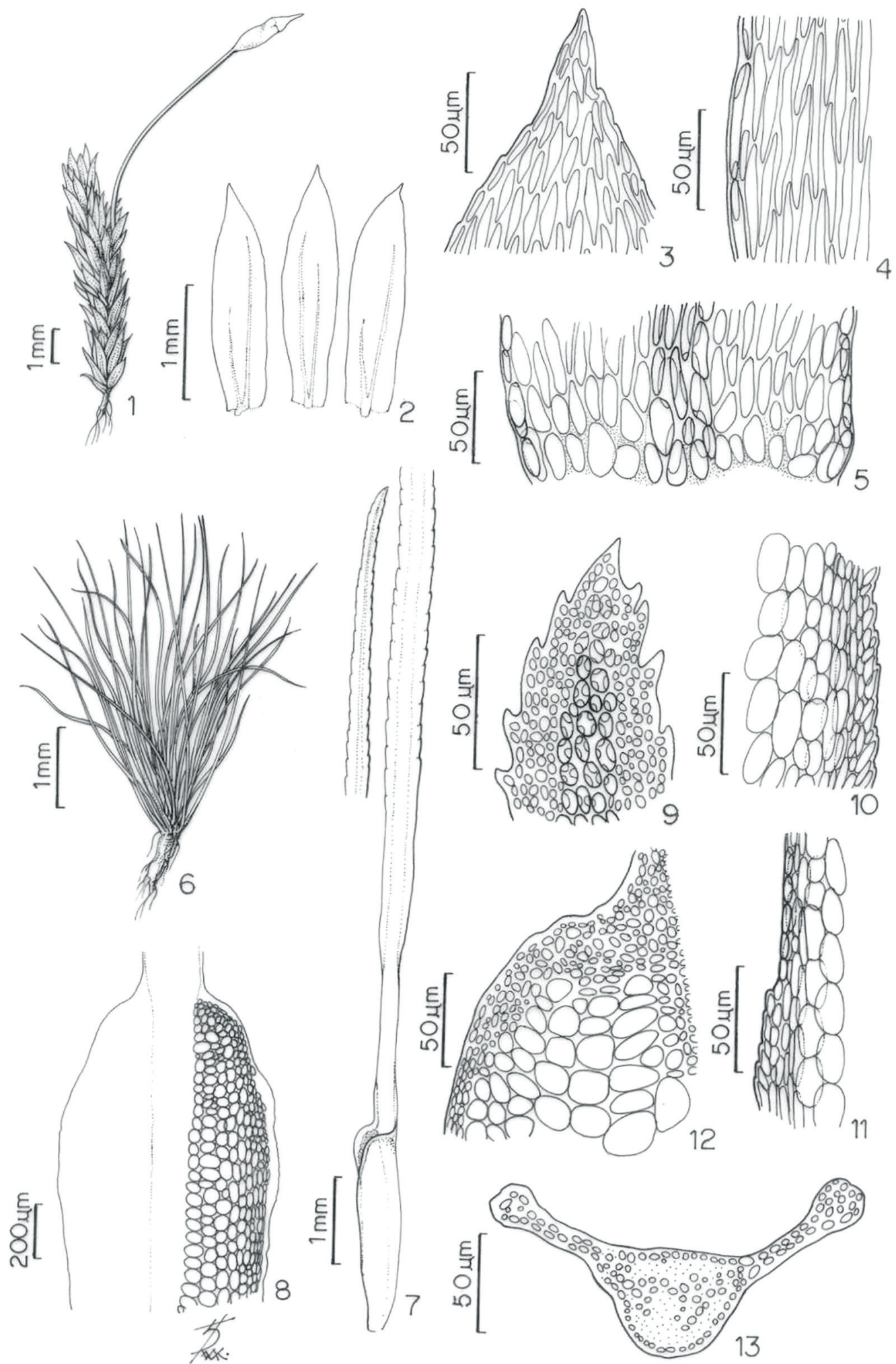

Figuras 1-5. Actinodontium integrifolium (Broth.) S.P. Churchill. 1. Aspecto geral da planta. 2. Filídios. 3. Detalhe do ápice do filídio. 4. Detalhe da margem na região mediana do filídio. 5. Detalhe da base do filídio. 6-13. Calymperes venezuelanum (Mitt.) Broth. ex Pittier 6. Aspecto geral do gametófito. 7. Filídio. 8. Porção inferior do filídio. 9. Ápice do filídio. 10-11. Detalhe da margem na porção basal do filídio. 12. Detalhe da porção superior do ombro. 13. Secção transversal do filídio na porção mediana. 
No levantamento florístico das hepáticas da área, $67 \%$ das espécies colonizaram troncos vivos. As folhas foram o segundo substrato mais colonizado (33\%), indicando o bom estado de preservação da floresta, visto que as epífilas são conhecidas pela exigência à alta umidade e sensibilidade a impactos destrutivos na estrutura da floresta. Troncos em decomposição representaram apenas $14 \%$ dos substratos colonizados (Valente \& Pôrto 2006b). A elevada representatividade de briófitas corticícolas em florestas tropicais tem sido observada em outros levantamentos (Germano \& Pôrto 2006; Molinaro \& Costa 2001). Por outro lado, muitas espécies não apresentaram exclusividade por substrato e colonizaram dois ou três tipos diferentes, o que é comum em áreas onde existe disponibilidade de substratos variados.

Considerando as faixas altitudinais referidas por Gradstein et al. (2001), a flora estudada inclui representantes de Florestas Tropicais de Terras Baixas (300/500 m), a exemplo dos gêneros Calymperes, Lepidopilum, Leucobryum, Syrrhopodon, e das espécies Pilosium chlorophyllum e Taxitelium planum, além de representantes de Florestas Tropicais Submontanas (300/500-1.000/1.400 m), como os gêneros, Phyllogonium e Thamniopsis e, até mesmo, elementos representantes de Florestas Tropicais Baixo Montana (1000/1400-2000/2500 m) como Squamidium, Thuidium e Zygodon.

Quando comparada a outras áreas de Mata Atlântica das regiões Nordeste e Sudeste do País, a flora de musgos do fragmento da Serra da Jibóia apresenta maior semelhança à dos estados de São Paulo (Visnadi 2005), e do Rio de Janeiro (Costa \& Lima 2005) com, respectivamente, $57 \%$ e $59 \%$ dos táxons em comum, e em menor proporção à de áreas do Estado de Pernambuco, 49\% das espécies compartilhadas (Pôrto 1990; 1992; Pôrto et al. 2004). Para o Estado da Bahia não se dispõe, até o momento, de publicações sobre levantamentos florísticos de musgos em remanescentes de Mata Atlântica.

A riqueza em musgos registrada neste trabalho, quando somada às 14 famílias, 41 gêneros e 70 espécies de hepáticas resultantes do levantamento realizado no mesmo remanescente da Serra da Jibóia (Valente \& Pôrto 2006a; 2006b), obtem-se um total de 37 famílias, 87 gêneros e 131 espécies. Sendo assim, este fragmento florestal apresenta brioflora consideravelmente rica, representando $24 \%$ do total de briófitas conhecido atualmente no Estado da Bahia. Esses números, aliados às peculiaridades da composição da brioflora assinaladas, reforçam a necessidade de preservação da biodiversidade da área, bem como dos demais remanescentes de Mata Atlântica existentes.

\section{Agradecimentos}

Ao Departamento de Ciências Biológicas da Universidade Estadual de Feira de Santana, pela disponibilidade de uso da infra-estrutura (Laboratório de Micologia - LAMIC e Herbário - HUEFS) fundamental para o desenvolvimento da pesquisa.

\section{Referências bibliográficas}

Bastos, C.J.P. \& Yano, O. 1993. Musgos da zona urbana de Salvador, Bahia, Brasil. Hoehnea 20: 23-33.

Bastos, C.J.P \& Yano, O. 2006. Briófitas de restinga das regiões Metropolitana de Salvador e Litoral Norte do Estado da Bahia, Brasil. Boletim do Instituto de Botânica 18: 197-205.

Bastos C.J.P.; Stradmann, M.T. \& Vilas Bôas-Bastos, S.B. 1998a. Additional contribution to the bryophyte flora from Chapada Diamantina National Park, State of Bahia, Brazil. Tropical Bryology 15: 15-20.

Bastos C.J.P.; Albertos, B. \& Vilas Bôas, S.B. 1998b. Bryophytes from some caatinga areas in the State of Bahia (Brazil). Tropical Bryology 14: 69-75.

Bastos, C.J.P. \& Villas Bôas-Bastos, S.B. 1998. Adições à brioflora (Bryopsida) do Estado da Bahia, Brasil. Tropical Bryology 15: 111-116.

Bastos, C.J.P.; Yano, O. \& Vilas Bôas-Bastos, S.B. 2000. Briófitas de campos rupestres da Chapada Diamantina, Estado da Bahia, Brasil. Revista Brasileira de Botânica 23: 357-368.

Buck, W.R. 1998. Pleurocarpous Mosses of the West Indies. Memoirs of The New York Botanical Garden 1: 1-401.

Campelo, M.J.A. \& Pôrto, K.C. 2007. Briófita epífita e epífila da RPPN Frei Caneca, Jaqueira, PE, Brasil. Acta Botanica Brasilica 21: 185-192

Costa, D.P. 1986. Leucobryaceae do Parque Nacional da Tijuca, Estado do Rio de Janeiro (Brasil). Rodriguésia 64/66: 41-48.

Costa, D.P. \& Lima, F.M. 2005. Moss diversity in the tropical rainforest of Rio de Janeiro, southeastern Brazil. Revista Brasileira de Botânica 28: 671-685.

Florschütz, P.A. 1964. The mosses of Suriname. Leiden, E.J. Brill.

Frahm, J.-P. 1991. Dicranaceae: Campylopodioideae, Paraleucobryoideae. Flora Neotropica, monograph 54: 1-237.

Germano, S.R. \& Pôrto, K.C. 2006. Bryophyte communities in an Atlantic forest remnant, state of Pernambuco, Brazil. Cryptogamie, Bryologie 27: 153-163.

Goffinet, B. \& Buck, W.R. 2004. Systematics of the Bryophyta (Mosses): From molecules to a revised classification. Pp. 205-239. In: B. Goffinet; V. Hollowell \& R. Magill (eds.). Molecular Systematics of Bryophytes. St. Louis, Missouri Botanical Garden.

Gradstein, S.R. \& Pócs, T. 1989. Bryophytes. Pp. 311-325. In: H. Lieth \& M.J.A. Werger (eds.). Tropical Rain Forest Ecosystems. Amsterdam, Elsevier Science Publischers B.V.

Gradstein, S.R.; Churchil, S.P. \& Salazar Allen, N. 2001. Guide to the bryophytes of Tropical America. Memories of The New York Botanical Garden 86: 1-577.

MMA. 2002. Biodiversidade Brasileira: Avaliação e Identificação de Áreas e Ações Prioritárias para Conservação, Utilização Sustentável e Repartição de Benefícios da Biodiversidade Brasileira. Brasília, Ministério do Meio Ambiente.

Molinaro, L.C. \& Costa, D.P. 2001. Briófitas do arboreto do Jardim Botânico do Rio de Janeiro. Rodriguésia 81: 107-124.

Peralta, D.F. \& Yano, O. 2006. Novas ocorrências de Musgos para o Estado de São Paulo, Brasil. Revista Brasileira de Botânica 29: $49-65$. 
Pôrto, K.C. 1990. Bryoflore d'une forêt de plaine et d'une forêt d'altitude moyenne dans l'État de Pernambuco - Brésil. Analyse floristique. Cryptogamie, Bryologie Lichénologie 11: $109-161$

Pôrto, K.C. 1992. Bryoflore d'une forêt de plaine et d'une forêt d'altitude moyenne dans l'État de Pernambuco (Brésil) 2. Analyse écologique comparative des Forêts. Cryptogamie, Bryologie Lichénologie 13: 187-219.

Pôrto, K.C.; Germano, S.R. \& Borges, G.M. 2004. Avaliação dos Brejos de altitude de Pernambuco e Paraíba, quanto à diversidade de Briófitas, para a Conservação. Pp.79-98. In: K.C. Pôrto; J.J.P. Cabral; M. Tabarelli (eds.). Brejos de Altitude em Pernambuco e Paraíba: história natural, ecologia e conservação. Brasília, Ministério do Meio Ambiente.

Queiroz, L.P.; Sena, T.S.N. \& Costa, M.J.S.L. 1996. Flora vascular da Serra da Jibóia, Santa Terezinha-Bahia. I: O Campo Rupestre. Sitientibus 15: 27-40.

Reese, W.D. 1993. Calymperaceae. Flora Neotropica, monograph 58: $1-102$.

Richards, W.P. 1984. The ecology of tropical forest bryophytes. Pp. 1233-1270. In: R.M. Schuster (ed.). v.2. New Manual of Bryology. Japan, Hattori Botanical Laboratory.

Sharp, A.J.; Crum, H. \& Eckel, P. 1994. The moss flora of Mexico. Memoirs of The New York Botanical Garden 69: $1-1113$

Sobrinho, J.G. \& Queiroz, L.P. 2005. Composição florística de um fragmento de Mata Atlântica na Serra da Jibóia, Santa Terezinha, Bahia, Brasil. Sitientibus. Série Ciências Biológicas 5: 20-28.

Valente, E.B. \& Pôrto, K.C. 2006a. Novas ocorrências de hepáticas (Marchantiophyta) para o Estado da Bahia, Brasil. Acta Botanica Brasilica 20: 195-201.

Valente, E.B. \& Pôrto, K.C. 2006b. Hepáticas (Marchantiophyta) de um fragmento de Mata Atlântica na Serra da Jibóia, município de Santa Teresinha, BA, Brasil. Acta Botanica Brasilica 20 433-441.

Valente, E.B. \& Pôrto, K.C. 2006c. Briófitas de uma área de afloramento rochoso na Serra da Jibóia, município de Santa Teresinha, Bahia, Brasil. Boletim do Instituto de Botânica 18: 207-211.

Vaz, T.F. \& Costa, D.P. 2006a. Os gêneros Brymela, Callicostella, Crossomitrium, Cyclodictyon, Hookeriopsis, Hypnella e Trachyxiphyum (Pilotrichaceae, Bryophyta) no Estado do Rio de Janeiro, Brasil. Acta Botanica Brasilica 20: $955-973$.

Vaz, T.F. \& Costa, D.P. 2006b. Os gêneros Lepidopilidium, Lepidopilum, Pilotrichum e Thamniopsis (Pilotrichaceae, Bryophyta) no Estado do Rio de Janeiro, Brasil. Acta Botanica
Brasilica 20: 975-993

Veloso, H.P. \& Góes Filho, L. 1982. Fitogeografia brasileira: classificação fisionômica- ecológica da vegetação neotropical. Boletim técnico (Série vegetação) n. 1, Salvador, IBGE.

Vilas Bôas-Bastos, S.B. \& Bastos, C.J.P. 1998. Briófitas de uma área de cerrado no município de Alagoinhas, Bahia, Brasil. Tropical Bryology 15: 101-110.

Vilas Bôas-Bastos, S.B. \& Bastos, C.J.P. 2004. Notes on the ocurrence of Hypnella pallescens (Hook.) A. Jaeger (Bryophyta, Pilotrichaceae) in Bahia, Brazil. Acta Botanica Malacitana 29: $160-163$.

Visnadi, S.R. 2002. Meteoriaceae (Bryophyta) da Mata Atlântica do Estado de São Paulo. Hoehnea 29: 159-188.

Visnadi, S.R. 2005. Brioflora da Mata Atlântica do Estado de São Paulo: região norte. Hoehnea 32: 215-131.

Visnadi, S.R. 2006. Sematophyllaceae da Mata Atlântica do nordeste do Estado de São Paulo. Hoehnea 33: 455-484.

Yano, O. 1981. A Checklist of Brazilian Mosses. The Journal of the Hattori Botanical Laboratory 50: 279-456.

Yano, O. 1989a. An Additional Checklist of Brazilian the Bryophytes. The Journal of the Hattori Botanical Laboratory 66: 371-434.

Yano, O. 1989b. Briófitas. Pp. 27-30. In: O. Fidalgo \& V.L.R. Bononi (eds.). Técnicas de coleta, preservação e herborização de material botânico. Série Documentos/Instituto de Botânica, São Paulo.

Yano, O. 1995. A New Additional Annotated Checklist of Brazilian Bryophytes. The Journal of the Hattori Botanical Laboratory 78: $137-182$.

Yano, O. 1996a. A checklist of the Brazilian Bryophytes. Boletim do Instituto de Botânica 10: 47-232.

Yano, O. 1996b. Criptógamos do PEFI, São Paulo, SP. Briófitas, 1: Mniaceae, Rhizogoniaceae, Racopilaceae, Phyllogoniaceae e Leucobryaceae (Bryales). Hoehnea 23: 81-98.

Yano, O. 2004. Novas ocorrências de briófitas para vários estados brasileiros. Acta Amazonica 34: 559-576.

Yano, O. 2006a. Novas adições ao catálogo de briófitas brasileiras. Boletim do Instituto de Botânica 17: 1-42.

Yano, O. 2006b. Novas adições as briófitas brasileiras. Boletim do Instituto de Botânica 18: 229-233.

Yano, O. \& Bastos, C.J.P. 1994. Musgos do Estado da Bahia, Brasil. Biologica Brasilica 6: 9-26.

Yano, O. \& Oliveira-e-Silva, M.I.M.N. 1997. Criptógamos do Parque Estadual das Fontes do Ipiranga, São Paulo, SP. Briófitas, 2: Fissidentaceae (Bryales). Hoehnea 24: 107-114

Yano, O. \& Pôrto, K.C. 2006. Diversidade das briófitas das matas serranas do Ceará, Brasil. Hoehnea 33: 7-39. 